\title{
A lady with Psycho-affective symptoms due to Hashimoto's encephalopathy
}

Jawad Bashir 1, Tamar Saeed 2, Irfan Khan 3, David Price1

1 Department of Diabetes \& Endocrinology, Morriston Hospital, Swansea, UK

2 Department of Diabetes \& Endocrinology, Royal Berkshire Hospital, Reading, UK ${ }^{3}$ Musgrove Park Hospital, Taunton, UK

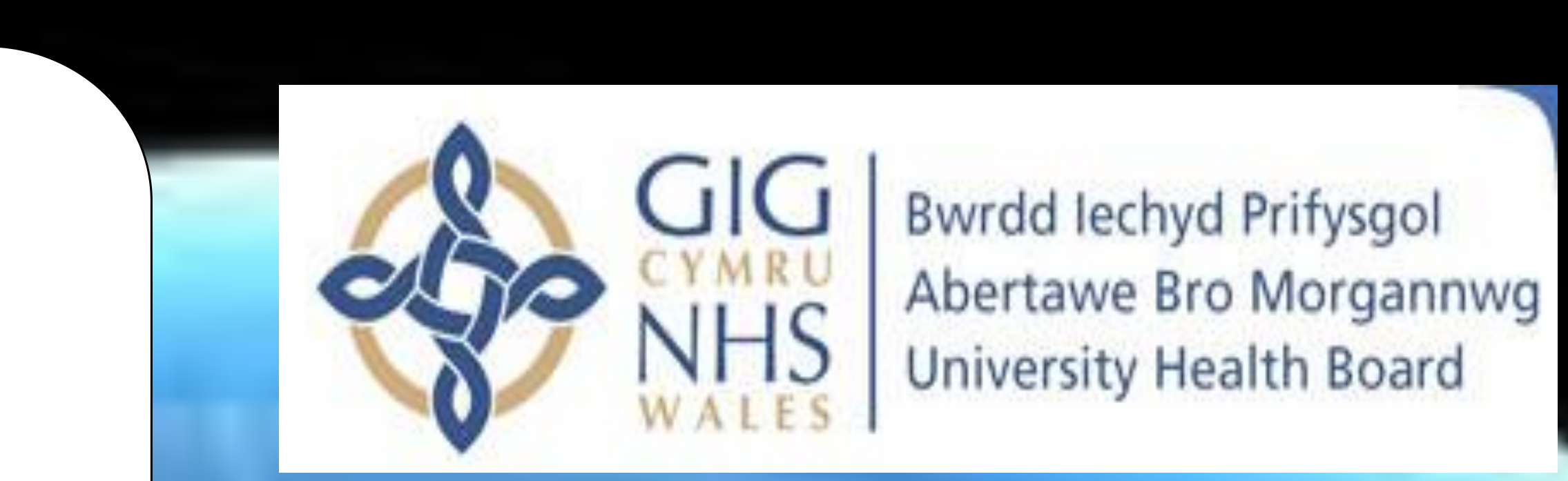

Introduction

Hashimoto's encephalopathy (HE) is a very rare neuropsychiatric condition associated with autoimmune thyroid disorders which shows a remarkable response to steroid therapy and hence is also called steroid-responsive encephalopathy associated with autoimmune thyroiditis (SREAT). It can be a diagnostic dilemma and we present a similar case presenting twice with psychoaffective symptoms but not receiving the diagnosis on first presentation.

Case Report
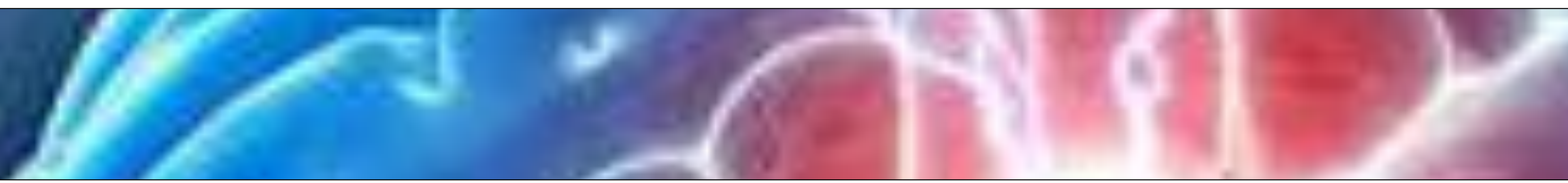

First presentation: A 44 year old lady presenting initially with 3 weeks history of low moods, lethargy, apathy and increased somnolence. She had a past medical history of well controlled hypothyroidism and epilepsy but no psychiatric problems. All the initial investigations including blood cultures, virology screen, CT brain and CSF analysis were reported as normal. She was labelled as being depressed and was discharged for outpatient FU with psychiatry team.

Second presentation: Two weeks later she re-presented with worsening symptoms of low mood, confusion and paranoid behaviour. O/E she was completely confused and showing awkward behaviour like spitting out her tablets, speaking alien language and was unable to follow simple commands. Previously she was living on her own with her daughter on a first floor flat but now was requiring assistance in all activities of life. There were no systemic features of sepsis.

Investigations

All test results were reported as normal including confusion screen tests (FBC, U\&E, Calcium, LFT, B12, folate, ferritin, Thyroid profile (TSH=1.6, FT4=19.6), CT head). Further tests requested after neurology review were as follows:

- Repeatedly negative blood cultures and virology screen

- Clear drug toxicology screen with undetectable ammonia and alcohol levels

- CSF analysis (Glucose=4.3 mmol/L; Protein=0.26 g/L; no xanthochromia; cultures/virology PCR -ve, no oligoclonal bands)

- Anti VGCC antibodies, anti VGKC antibodies, anti NMDA antibodies also NOT detected

- Her anti-TPO antibodies were strongly positive with very high titres (>600 IU $/ \mathrm{ml}$ ) - The MRI brain showed non specific T2 hyper-intense signal in deep white matter EEG showed non specific slowing of background activity

The MRI and EEG findings suggested metabolic/toxic encephalopathy.

It was only after excluding all the metabolic and infective encephalopathies, the final diagnosis of Hashimoto's encephalopathy was reached. She was started on high dose of oral steroids (60 $\mathrm{mg}$ prednisolone daily) tapered down slowly over 2 months while in neurorehabilitation. There was immediate improvement in neuropsychiatric symptoms but she still continues to have emotional lability.

Discussion

Hashimoto's encephalopathy (HE) doesn't have direct relationship with thyroid status but elevated anti TPO antibodies are essential diagnostic feature suggesting autoimmune thyroid disease. Majority (75\%) present with psycho-affective features while the remaining (25\%) behave like stroke with focal neurology. The prognosis of cognitive decline is variable with residual neuropsychiatric features. Steroids and optimization of thyroid status are the mainstay of treatment with $98 \%$ response rate in acute setting but relapses are common.

diagnosis of exclusion which is often missed but if considered after ruling out other causes of such presentations, it shows dramatic response to steroids. Occasionally patients need steroid sparing drugs in case of no response.

This case illustrates that HE can present with psychiatric symptoms in patients with long standing well- controlled hypothyroidism. 